individuals at risk for psychosis and bipolar disorder. Schizophr Res. 2015;168:50-5.

2 Zanini MA, Castro J, Cunha GR, Asevedo E, Pan PM, Bittencourt L, et al. Abnormalities in sleep patterns in individuals at risk for psychosis and bipolar disorder. Schizophr Res. 2015;169:262-7.

3 Lunsford-Avery JR, LeBourgeois MK, Gupta T, Mittal VA. Actigraphicmeasured sleep disturbance predicts increased positive symptoms in adolescents at ultra high-risk for psychosis: a longitudinal study. Schizophr Res. 2015;164:15-20.

4 Zanini M, Castro J, Coelho FM, Bittencourt L, Bressan RA, Tufik S, et al. Do sleep abnormalities and misaligned sleep/circadian rhythm patterns represent early clinical characteristics for developing psychosis in high risk populations? Neurosci Biobehav Rev. 2013;37:2631-7.

5 Gonçalves BS, Adamowicz T, Louzada FM, Moreno CR, Araujo JF. $A$ fresh look at the use of nonparametric analysis in actimetry. Sleep Med Rev. 2015;20:84-91.

\section{Anorexia nervosa presenting as a subacute sensory-motor axonal polyneuropathy}

Rev Bras Psiquiatr. 2016;38:179-180

doi:10.1590/1516-4446-2015-1846

Anorexia nervosa is a psychiatric disorder characterized by abnormally low body weight associated with fear of gaining weight, body image disturbance, and restriction of caloric intake. It is categorized as restrictive when accompanied by a reduction in calorie consumption; or as purging when there is intentional overuse of diuretics, laxatives, or self-induced vomiting. Anorexia affects 0.3 to $0.9 \%$ of young women and $0.3 \%$ of young men. ${ }^{1}$

Despite the knowledge regarding the deleterious effects of anorexia nervosa on several organ systems, ${ }^{2}$ neurological complications are still under-recognized. Only a few case reports so far have linked anorexia nervosa to brain atrophy ${ }^{3}$ and peripheral neuropathy. ${ }^{4,5}$

Here we report the case of a young woman with subacute symptoms of peripheral polyneuropathy. Clinical and laboratory work-up identified vitamin deficiency secondary to hitherto undiagnosed anorexia nervosa.

A 24-year-old woman reported burning pain in her feet for three months. Two months after the onset of dysesthesia, she gradually developed a paralysis of the lower limbs. At this point, she had an orthopedic consultation which ruled out osteoarticular disease. Due to the worsening of motor and sensitive symptoms, she looked for medical assistance at the emergency department of the university hospital at Universidade Federal de Minas Gerais, Belo Horizonte, Brazil. She denied any previous disease and use of drugs.

Physical examination showed a very thin woman with body mass index of $14.9 \mathrm{~kg} / \mathrm{m}^{2}$. Neurological examination revealed absence of the deep reflexes in the lower limbs along with paraparetic gait and ataxia.

Laboratory workup did not reveal any abnormalities in complete blood count, electrolyte levels, renal, hepatic, and thyroid functions, and thiamine. Reduced levels of folate (2.05 nmol/L; normal values: > $5.9 \mathrm{nmol} / \mathrm{L})$ and vitamin B12 (190 pg/mL; 200-900 pg/mL) were detected. Electromyography confirmed the presence of symmetric sensory-motor axonal polyneuropathy. No change in gastric mucosa was evidenced by upper digestive endoscopy with biopsy.

Since the patient denied diarrhea or other symptoms that could indicate a malabsorption syndrome, a comprehensive approach tried to characterize her dietary habits. She claimed to be vegetarian, with occasional consumption of eggs and milk derivatives. She was afraid of gaining weight, and over the past three years persistently felt overweight. Moreover, she had morning episodes of vomiting after breakfast, and consumed progressively less food. The diagnosis of anorexia nervosa, restrictive subtype, was established.

Her vitamin deficiency was treated with intramuscular cyanocobalamin and oral vitamin B complex plus folic acid supplementation. Gabapentin (900 mg/day) was used for neuropathic pain. On follow-up she evolved with complete remission of sensory and motor symptoms, and recovery of normal weight and change of dietary habits under psychiatric and dietitian supervision.

The association between anorexia nervosa and peripheral neuropathy has rarely been reported. ${ }^{4,5} \mathrm{~A}$ unique feature of the present case was the diagnosis of anorexia nervosa only after clinical and laboratory investigation of polyneuropathy. Until then, her neurological symptoms and weight loss were attributed to an undefined clinical condition. The precise diagnosis prevented unnecessary investigation, making clinical improvement possible.

Long periods of restricted food intake along with frequent vomiting may promote significant reduction of vitamin B12 acquisition. These facts, associated with the patient's vegetarian diet contributed to the development of this peculiar clinical presentation. Moreover, the absence of abnormalities on upper digestive endoscopy followed by histological analysis excluded the presence of local diseases that could justify B12 hypovitaminosis (e.g., atrophic gastritis). Clinical recovery with cyanocobalamin administration corroborates the causal assumption proposed. It should be mentioned that treatment of a case such as the one we described cannot be limited to vitamin supplementation and/or relieving of dysesthesia. Prompt weight restoration under dietary and psychiatric supervision is also essential. The severity of physical symptoms associated with anorexia nervosa possibly contributed to the patient's adherence to treatment and/or subsequent clinical improvement. Interestingly enough, a positive outcome regarding the eating disorder has also been reported for a severe case of anorexia nervosa presenting with peripheral neuropathy and Wernicke encephalopathy. ${ }^{5}$

The present case report highlights an atypical and severe form of anorexia nervosa. Early recognition and treatment of this illness might have prevented unnecessary neurological complications. Eating disorders must be considered in the differential diagnosis of vitamin deficiency syndromes. 
Antonio L. Teixeira, ${ }^{1,2,3}$ Bruno T. Junho, ${ }^{3}$ João L. Barros, ${ }^{3}$ Rodrigo S. Gomez ${ }^{2}$

${ }^{1}$ Grupo de Neuropsiquiatria, Serviço de Neurologia, Hospital das Clínicas, Universidade Federal de Minas Gerais (UFMG), Belo Horizonte, MG, Brazil. ${ }^{2}$ Ambulatório de Doenças Neuromusculares, Servico de Neurologia, Hospital das Clínicas, UFMG, Belo Horizonte, MG, Brazil. ${ }^{3}$ Laboratório Interdisciplinar de Investigação Médica, Faculdade de Medicina, UFMG,

Belo Horizonte, MG, Brazil

Submitted Nov 23 2015, accepted Dec 042015.

\section{Disclosure}

The authors report no conflicts of interest.

\section{References}

1 Hudson Jl, Hiripi E, Pope HG Jr, Kessler RC. The prevalence and correlates of eating disorder in the National Comorbidity Survey Replication. Biol Psychiatry. 2007;61:348-58.

2 Mehler PS, Brown C. Anorexia nervosa - medical complications. J Eat Disord. 2015;3:11.

3 Kraeft JJ, Uppot RN, Heffess AM. Imaging findings in eating disorders. AJR Am J Roentgenol. 2013;200:W328-35.

4 Cerutti D, Guilpain P, Schiffmann A, Rivière S, Carra C, Labauge P, et al. Anorexie psychogène et carences vitaminiques sévères dans les suites d'une gastrectomie longitudinale. Rev Med Interne. 2014;35:609-12.

5 Renthal W, Marin-Valencia I, Evans PA. Thiamine deficiency secondary to anorexia nervosa: an uncommon cause of peripheral neuropathy and Wernicke encephalopathy in adolescence. Pediatr Neurol. 2014;51:100-3. 